\title{
The prognostic value and immunological role of the small mother against decapentaplegic proteins in kidney renal clear cell carcinoma
}

\author{
Siyue Zhang ${ }^{1,2,3,4 \#}$, Zhuang Liu ${ }^{3,4 \#}$, Mingming Xiao ${ }^{3,4 \#}$, Hong Liu ${ }^{1,2}$ \\ ${ }^{1}$ The Second Department of Breast Tumor Surgery, Tianjin Medical University Cancer Institute and Hospital, National Clinical Research Center \\ for Cancer, Key Laboratory of Cancer Prevention and Therapy, Tianjin, China; ${ }^{2}$ Tianjin's Clinical Research Center for Cancer, Tianjin, China; \\ ${ }^{3}$ Department of Biochemistry and Molecular Biology, Tianjin Medical University Cancer Institute and Hospital, National Clinical Research Center \\ for Cancer, Key Laboratory of Cancer Prevention and Therapy, Tianjin, China; ${ }^{4}$ Tianjin's Clinical Research Center for Cancer, Key Laboratory of \\ Breast Cancer Prevention and Therapy, Tianjin Medical University, Ministry of Education, Tianjin, China \\ Contributions: (I) Conception and design: S Zhang, Z Liu, H Liu; (II) Administrative support: H Liu; (III) Provision of study materials or patients: \\ H Liu; (IV) Collection and assembly of data: S Zhang; (V) Data analysis and interpretation: Z Liu, S Zhang, M Xiao; (VI) Manuscript writing: All \\ authors; (VII) Final approval of manuscript: All authors. \\ "These authors contributed equally to this work. \\ Correspondence to: Hong Liu, MD, PhD. Tianjin Medical University Cancer Institute and Hospital, West Huanhu Road, Tianjin 300060, China. \\ Email: liuhong_submit@tjmuch.com.
}

Background: The small mother against decapentaplegic proteins (SMADs) are a family of transforming growth factor (TGF)- $\beta$ signal transduction molecules, playing a vital role in the initiation and development of tumors. This research aimed to determine SMADs' prognostic values and their involvement in immune infiltration.

Methods: Expression patterns and prognostic values of SMADs were evaluated by pan-cancer analysis in multiple cancer cohorts based on The Cancer Genome Atlas data. cBioPortal database was used for genetic mutation analyses. UALCAN and LinkedOmics databases were applied for the analysis of the methylation level and its correlation with gene expression, respectively. The correlation of gene expression was analyzed by Gene Expression Profiling Interactive Analysis platform. Additionally, we utilized the Tumor Immune Estimation Resource database to explore the correlation between SMAD expressions and the number of tumor-infiltrating immune cells. Functional prediction was performed by Gene Set Enrichment Analysis (GSEA) method.

Results: We found that the expressions of SMAD1, 2, 3, 4, 6 were significantly decreased whereas the expression of SMAD9 was significantly increased in kidney renal clear cell carcinoma (KIRC) tissues than in normal control tissues. And aberrant DNA methylation in the promoter regions may cause the dysregulation of these differentially expressed SMADs. Also, we found that the expressions of SMAD1, 2, 3, 4, 6 decreased significantly with the progression of KIRC tumors, and their high expression level was significantly associated with favorable prognoses of KIRC patients. Genetic mutations analysis using the cBioPortal database found that there were missense mutations in SMAD2 and 4, and truncation mutations in SMAD2 and 3. Further, SMAD1, 2, 3, 4, 6 expressions showed correlations with diverse immune infiltrating cells and immune markers. In particular, SMAD1, 2, 4 expressions were strongly correlated with monocyte, tumor-associated macrophage, M1/M2 macrophage, revealing their potential to regulate the polarity of macrophages. Finally, function prediction by GSEA indicated that SMAD1, 2, 3, 4, 6 were closely involved in immune-related signaling pathways.

Conclusions: Our findings indicate that SMAD1, 2, 3, 4 and 6 were potent biomarkers for predicting the prognosis and immune cell infiltration of KIRC patients. 
Keywords: Small mother against decapentaplegic protein (SMAD); kidney renal clear cell carcinoma (KIRC); expression patterns; prognosis; immune infiltration

Submitted Jan 27, 2021. Accepted for publication May 10, 2021.

doi: $10.21037 /$ tcr-21-178

View this article at: https://dx.doi.org/10.21037/tcr-21-178

\section{Introduction}

Renal cell carcinoma (RCC), present in the epithelium of renal tubules, accounts for more than $85 \%$ of all renal malignancies and $2 \%$ of the global cancer burden. Kidney renal clear cell carcinoma (KIRC), which comprises about $70 \%$ to $80 \%$ of RCC, is the most common histological subtype and a major cause of cancer-related deaths $(1,2)$. Tumor recurrence occurs in approximately $30-40 \%$ of KIRC patients after curative treatment $(2,3)$. Despite the fast development of novel approaches to combating KIRC (4), less than $10 \%$ of patients with metastatic KIRC can survive for 5 years after diagnosis (1). Therefore, it is urgently needly to explore potential new prognostic biomarkers or therapeutic targets, to improve the diagnosis, prognosis and individualized treatment of KIRC patients.

Transforming growth factor (TGF)- $\beta$ family signaling regulates cell proliferation and carcinogenesis via activating the canonical small mother against decapentaplegic (SMAD) dependent as well as the non-canonical SMAD independent pathways (5). The SMAD family comprises transcription factors that mediate the diverse effects of the TGF- $\beta$ superfamily (6). Eight members of the SMAD family exist in vertebrates, which can be divided into three different subgroups according to their distinct functions in TGF- $\beta$ signal transduction: receptorregulated SMADs (SMAD1, 2, 3, 5, 9), common mediator SMAD4, and inhibitory SMADs (SMAD6, 7) $(7,8)$. Studies have suggested that abnormalities of SMAD-related signaling pathways are involved in tumorigenesis in some malignancies, such as cancers of the colon, lung, pancreas, etc. (9-11). Abnormal expression of SMADs has been well documented, and related studies have demonstrated that some SMADs may also be used as prognostic biomarkers in some cancers $(12,13)$. However, the expression of SMADs family genes in patients with KIRC and their prognostic significance is still elusive. Thus, we hypothesized that some genes of SMAD could also have an important prognostic role in KIRC.

Immune cells infiltrated into tumors form a complex ecosystem in the surrounding microenvironment to control tumor development and are closely correlated with the clinical consequences of various cancers including KIRC
(14-16). Tumor immune infiltrating cells are generally classified as adaptive immune cells ( $\mathrm{T}$ and B lymphocytes) and innate immune cells [e.g., macrophages, neutrophils and dendritic cells (DCs)]. These immune cells with different functions communicate with each other directly or indirectly, and jointly affect the growth of tumor cells (17). In recent years, vigorous development of immunotherapy interventions has greatly improved the clinical outcomes in patients with KIRC (18). Therefore, immune infiltrating cells are considered promising targets for KIRC therapy.

However, few studies have deciphered the expression pattern, prognostic value, and immune infiltrating relevance of SMAD family genes in KIRC. The aims of this research were to determine SMADs' prognostic values and their involvement in immune infiltration. In this study, we performed an integrated and systematic analysis including the expression pattern, prognostic value, and immune infiltrating relevance of SMAD family genes in KIRC by using The Cancer Genome Atlas (TCGA)-based gene expression data and online public platforms, and further explored their potential functions.

We present the following article in accordance with the REMARK reporting checklist (available at https://dx.doi. org/10.21037/tcr-21-178).

\section{Methods}

\section{TCGA and cBioPortal database analysis}

RNA-Seq data of KIRC patients were obtained from the TCGA database (https://portal.gdc.cancer.gov/), which was extracted for gene expression analysis and Gene Set Enrichment Analysis (GSEA) method. The data of SMADs genetic mutation in KIRC (TCGA, firehose legacy) were extracted from cBioPortal (https://www.cbioportal.org). Overall survival (OS) and disease-free survival (DFS) were set as the clinical endpoint to evaluate the role of SMADs in KIRC. OS means the estimation of time from start of treatment to death due to any cause. DFS indicates the percentage of people who are alive for a given period of time after diagnosis. The study was conducted in accordance 
with the Declaration of Helsinki (as revised in 2013).

\section{Methylation analysis in UALCAN}

UALCAN (http://ualcan.path.uab.edu) is a publicly available and powerful web portal for in-depth analysis of cancer OMICS data (TCGA and MET500) (19). In our study, the methylation level of SMADs was analyzed in KIRC by the "TCGA" dataset.

\section{LinkedOmics}

LinkedOmics (http://www.linkedomics.org/login.php), an easy-to-use and publicly available portal, provides an interactive platform for clinicians and biologists to obtain, compare and analyze tumor multi-omics data across 32 TCGA tumor types (20). We used the Linked Finder module to analyze the correlation of gene expression and methylation level of SMADs in KIRC. Pearson correlation coefficient was used in this analysis.

\section{Gene correlation analysis in Gene Expression Profiling Interactive Analysis (GEPIA)}

GEPIA (http://gepia.cancer-pku.cn/) is an online database based on data from 8,587 normal tissues and 9,736 tumors from TCGA and the GTEx Project (21). In our study, GEPIA was applied to analyze SMAD gene expression and prognostic value in KIRC. And correlation analysis of gene expression was performed with the "correlation analysis" module in GEPIA. Correlation analysis was performed with Spearman correlation coefficient.

\section{Protein-protein interaction (PPI) network analysis}

To understand the interaction relationship and molecular mechanism among differentially expressed SMADs, we constructed PPI networks using the STRING database (https://string-db.org/) and the GeneMANIA database (http://genemania.org).

\section{Immune infiltration analysis in Tumor Immune Estimation Resource (TIMER)}

TIMER database (https://cistrome.shinyapps.io/timer/) is used for analyzing immune infiltration based on RNA sequencing data from a variety of tumors (22). We explored the connection between the expressions of SMADs and six tumor-infiltrating immune cells (B cells, $\mathrm{CD}^{+} \mathrm{T}$ and $\mathrm{CD} 8^{+}$ $\mathrm{T}$ cells, neutrophils, macrophages, and DCs). We also explored the expression correlation between differentially expressed SMAD genes and marker genes of immune cells, including tumor-associated macrophage (TAM), M1/M2 macrophage, DCs, neutrophils, Th1, Th2, Treg and natural killer cells through relevant modules. We specifically assessed the correlation between differentially expressed SMADs and macrophage polarity through the "correlation" module, and macrophage related markers are as follows: CD86/CSF1R for monocyte, IL10/VSIG4/PCGR2A for TAM, NOS2/PTGS2 for M1 macrophages and CD163/ NS4A4A/MRC1 for M2 macrophages. Correlation analysis was performed with Spearman correlation coefficient.

\section{GSEA}

GSEA focuses on groups of genes that share biological function, common regulation or chromosomal location (23). In this study, GSEA was applied to explore the potential regulatory mechanisms of SMADs expression of KIRC (TCGA).

\section{Statistical analysis}

GraphPad Prism 6.0 or SPSS Version 20 software was used to perform the statistical analyses in our study. The Student's $t$-test was used to determine the statistical difference in the two groups. The correlation of gene expression (GEPIA) and the connection between gene expression and immune cell infiltration or immune cell marker genes (TIMER) was evaluated by statistical significance as well as Spearman's correlation.

\section{Results}

The expression pattern and methylation level of SMAD family genes in KIRC patients

Firstly, we used 530 KIRC tissues and 72 normal control tissues, as well as 72 paired KIRC tissues and corresponding adjacent normal tissues in TCGA to evaluate the expression pattern of SMADs. Consistently, we found that the expressions of SMAD1, 2, 3, 4 and 6 were significantly reduced in KIRC tissues than in normal control tissues, while expression of SMAD9 was significantly increased in cancer tissues (Figure1A,B). And these differentially expressed SMADs were further verified in the GEPIA database (Figure $1 C, D, E, F, G, H$ ). DNA methylation, especially the excessive methylation of the promoters 
of tumor suppressor genes, can lead to transcriptional silencing, which has a crucial impact on the development of KIRC (24). In order to explore the methylation levels of these differentially expressed SMADs in KIRC, we used UALCAN to evaluate the methylation status of their promoter regions. As shown in Figure 1I, f, K,L, M,N, the promoters of SMAD1, 2, 3, 4, and 6 showed more methylation in KIRC tissues. Further, the correlation between expression patterns and the methylation status of SMADs was explored in KIRC. As presented in Figure 1O,P,Q,R,S,T, the expressions of all differentially expressed SMAD genes were significantly negatively correlated with their methylation levels. Altogether, these data manifested that the changes in the methylation level of the SMAD promoter regions may be an important cause for regulating the expression pattern of SMADs in KIRC.

The expression pattern of these SMADs was also assessed in the main tumor stages of KIRC patients. As shown in Figure 2, we found that the expressions of SMAD1, 2, 3, 4 and 6 decreased significantly with the progression of KIRC tumors. This suggested that expressions of these SMADs had significant impacts on the inhibition of tumorigenesis and progression of KIRC.

\section{The prognostic value of differential expression of SMAD genes in KIRC patients}

Next, we investigated the prognostic value of SMADs in KIRC patients. By drawing survival significance maps according to the Cox proportional hazard ratio (HR) using the GEPIA database, we evaluated whether differential expressions of these SMADs have impact on OS and DFS of KIRC patients. We found that low expressions of SMAD1, 2, 3, 4 and 6 were significantly associated with poor OS of KIRC patients (Figure $3 A$ ), and low expressions of SMAD2, 3, 4 and 6 were significantly associated with poor DFS of KIRC patients (Figure 3B). The Kaplan-Meier plots further showed that KIRC patients with decreased SMAD1, 2, 3, 4, and 6 expressions had shorter OS (Figure 3C,D,E,F,G), and KIRC patients with decreased SMAD2, 3, 4, 6 expressions had shorter DFS (Figure 3H,I,F,K). Since SMAD1, 2, 3, 4 and 6 had better prognostic value, the following analysis mainly focused on these 5 SMAD genes.

\section{Genetic mutations, expression correlation, and interaction network analyses of SMAD genes in KIRC patients}

Then, an overall exploration of the molecular properties of these $5 \mathrm{SMAD}$ genes was performed. The cBioPortal online tool was applied to assess the genetic mutations of these SMADs. In total, 538 KIRC patients (TCGA, firehose legacy) were selected. Figure $4 A$ exhibited that the missense mutations were found in SMAD2 and 4, and the truncating mutation was found in SMAD2 and 3. Next, the potential co-expression of SMADs was analyzed. We found that there was a moderate to high correlation among the expressions of SMAD1, 2, 3 and 4 (Figure 4B). Moreover, a PPI network analysis of these SMADs was conducted by the STRING database to assess the potential interactions among them. As expected, 5 nodes and 10 edges were obtained in the PPI network, indicating the close connection among these SMADs (Figure 4C). In addition, GeneMANIA's PPI network results further showed that the functions of these SMADs are mainly related to transmembrane receptor protein serine/threonine kinase signaling pathway, BMP signaling pathway, TGF- $\beta$ signaling pathway, SMAD protein complex assembly and SMAD binding, etc. (Figure 4D).

\section{SMAD1/2/3/4/6 expressions were correlated with immune cell infiltration in KIRC patients}

Infiltrating immune cells in the tumor microenvironment is critical to modulate KIRC progression and has shown promising prognostic value (15). We further utilized the TIMER database to explore the relevance between SMAD1, 2, 3, 4 and 6 expressions and immune infiltrating cells (including $\mathrm{B}$ cells, $\mathrm{CD} 8^{+} \mathrm{T}$ cells, $\mathrm{CD}^{+}{ }^{+} \mathrm{T}$ cells, macrophages, neutrophils, and DCs) in KIRC. Interestingly, we observed that SMAD1 expression was positively correlated with the infiltration of five immune cells except B cells (Figure 5A), and the expression levels of SMAD2, 3 and 4 were positively correlated with all infiltrating immune cells evaluated (Figure 5B,C,D). In addition, the expression of SMAD6 was positively correlated with the level of infiltrating $\mathrm{CD}^{+} \mathrm{T}$ and $\mathrm{CD}^{+} \mathrm{T}$ cells, while not significantly correlated with the levels of infiltrating B cells, macrophages, neutrophils and DCs (Figure 5E). These data suggested that the expressions of SMAD1, 2, 3, 4 and 6 were closely linked to the level of infiltration immune cells.

\section{Correlation analysis between SMAD1/2/3/4/6 expressions and immune markers}

To unravel the effects of the expressions of SMAD1, 2, 3, 4 and 6 on immune infiltration, we further analyzed the relationships between their expressions and multiple specific 
A

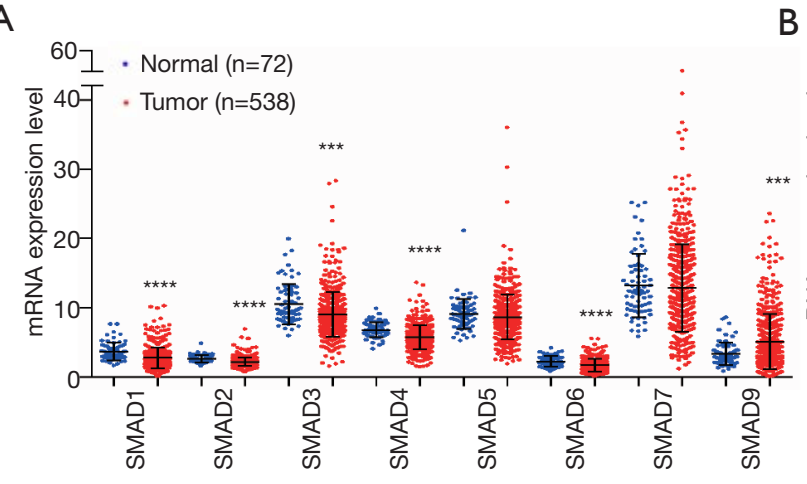

$B$
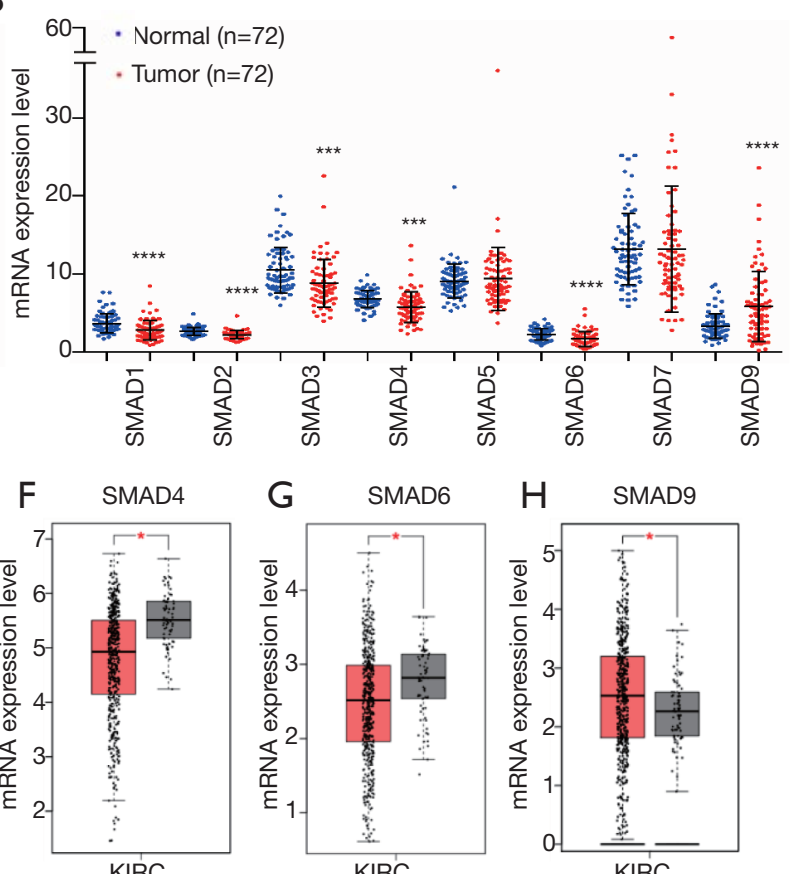

$n(T)=523, n(N)=72$

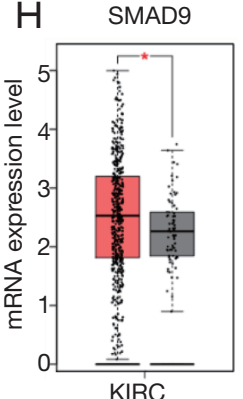

$n(T)=523, n(N)=72$
$n(T)=523, n(N)=72$

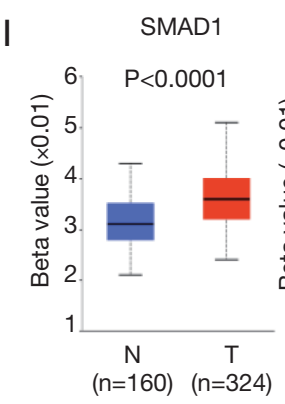

$n(T)=523, n(N)=72$

$n(T)=523, n(N)=72$
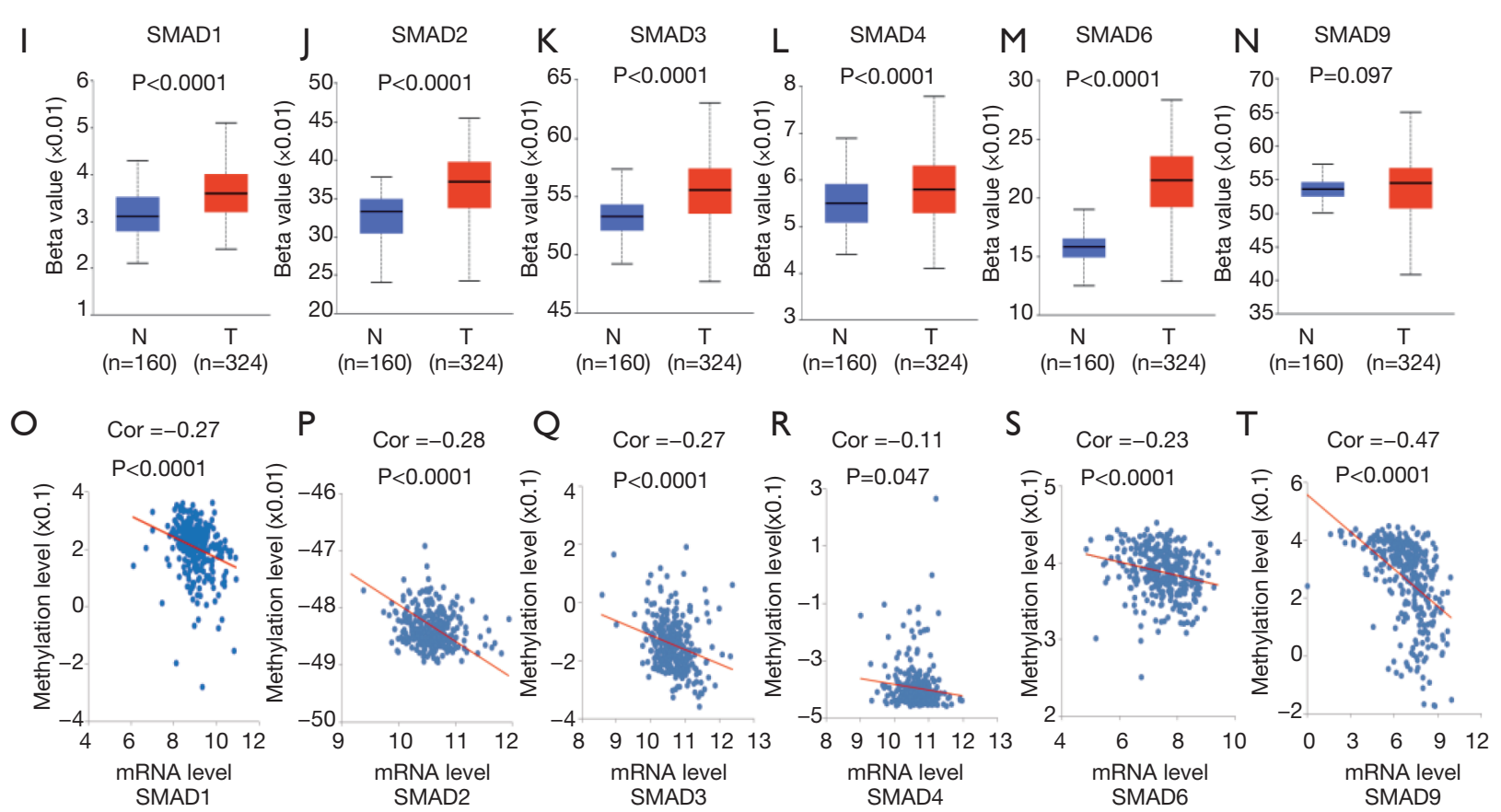

Figure 1 The expression pattern and methylation level of SMAD family genes in KIRC patients. (A,B) The expression patterns of SMAD1, 2, 3, 4, 5, 6, 7 and 9 in 530 KIRC patient tissues and 72 normal tissues as well as 72 paired KIRC tissues and corresponding adjacent normal tissues (TCGA). The expression patterns of SMAD1 (C), SMAD2 (D), SMAD3 (E), SMAD4 (F), SMAD6 (G), SMAD9 (H). Promoter methylation status of SMAD1 (I), SMAD2 (J), SMAD3 (K), SMAD4 (L), SMAD6 (M), SMAD9 (N) in 324 KIRC patient tissues and 160 normal tissues (UALCAN). The correlation between the expressions of SMAD1 (O), SMAD2 (P), SMAD3 (Q), SMAD4 (R), SMAD6 (S), SMAD9 $(\mathrm{T})$ with their promoter methylation status (LinkedOmics). $\mathrm{P}<0.05$ was considered significant $\left({ }^{*}, \mathrm{P}<0.05 ;{ }^{* * *}, \mathrm{P}<0.001 ;{ }^{* * * *}\right.$ $\mathrm{P}<0.001)$. SMAD, small mother against decapentaplegic protein; KIRC, kidney renal clear cell carcinoma. 
A

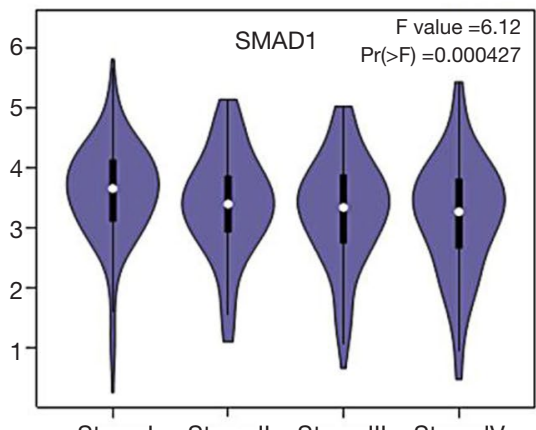

Stage I Stage II Stage III Stage IV

D

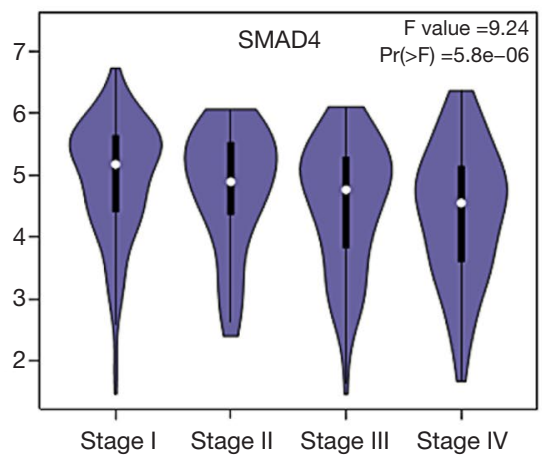

B

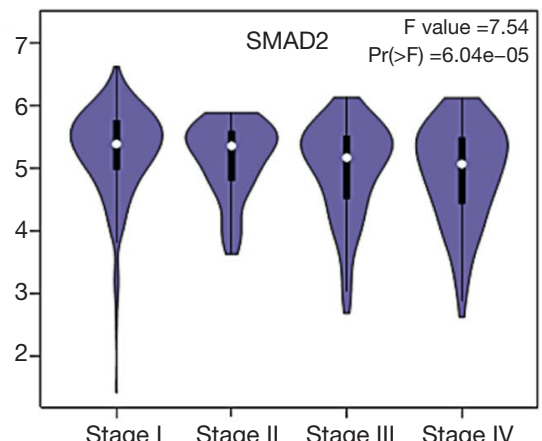

E

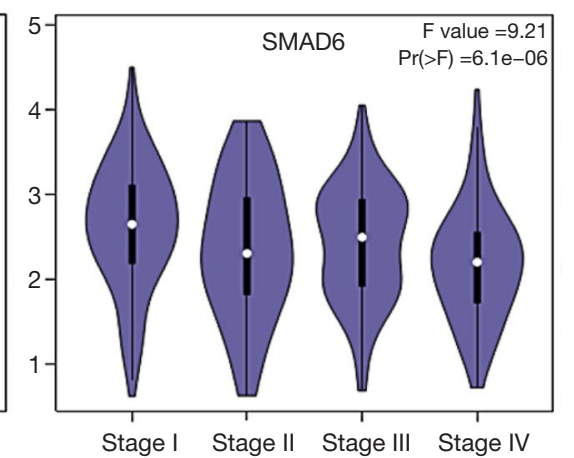

C

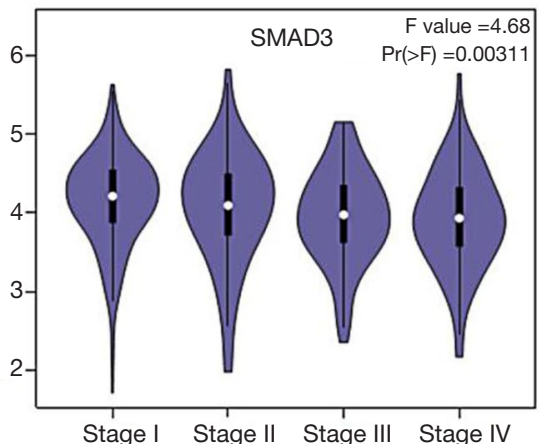

$\mathrm{F}$

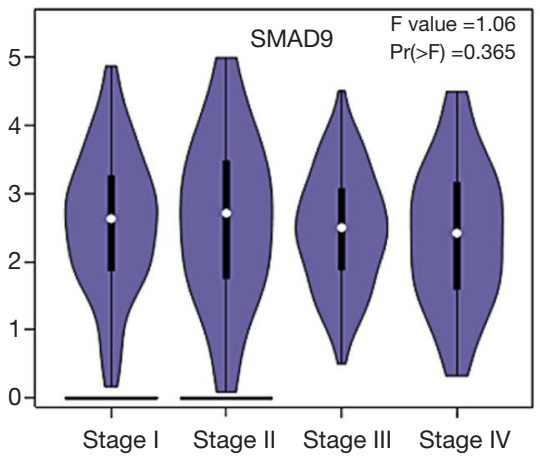

Figure 2 The expression pattern of SMAD genes in the main pathological stages of KIRC patients. The expression patterns of SMAD1 (A), SMAD2 (B), SMAD3 (C), SMAD4 (D), SMAD6 (E), SMAD9 (F) in the main pathological stages of KIRC patients. P<0.05 was considered significant. SMAD, small mother against decapentaplegic protein; KIRC, kidney renal clear cell carcinoma.

markers of innate and adaptive immune cells including monocyte, TAM, M1 macrophage, M2 macrophage, DCs, Th1, Th2, Treg, and natural killer cell in KIRC (Table 1). We found that SMAD1 and 4 expressions showed a significant correlation with the genetic markers of these infiltrating immune cells, and the expression of SMAD2 was correlated with most immune markers of infiltrating immune cells except for natural killer cells. In addition, the expression of SMAD3 had a significant correlation with immune markers of M1 macrophage, DCs and Th1, and the expression of SMAD6 was remarkably linked to the genetic markers of DCs, Treg and natural killer cells in KIRC. Particularly, we found that the expressions of SMAD1, 2 and 4 were strongly correlated with the levels of specific markers of monocytes, TAM, M1 and M2 macrophages in KIRC (Table 1 and Figure 6), which has also been further verified in the GEPIA database (Table 2). This suggested that SMAD2, 3, and 5 may be involved in regulating the polarity of macrophages in KIRC.

\section{Functional enrichment analysis of SMAD1/2/3/4/6 in patients with KIRC}

To better understand the regulatory functions of SMAD1, 2, 3, 4 and 6 in KIRC, the GSEA method was employed to explore their regulatory mechanisms. All samples were separated into the low group and the high group based on the mean value. The bubble diagrams (Figure 7) showed the signal pathways that were significantly enriched in the low expression groups of SMAD1, 2, 3, 4, and 6. We found that immune-related signaling pathways, including the primary immunodeficiency, antigen processing and presentation, natural killer cell-mediated cytotoxicity, and $T$ cell receptor signaling pathway, were significantly enriched and negatively correlated with SMAD1, 2, 3, 4 and 6. Also, SMAD1, 2, 3, 4 and 6 were closely related to cytokine-cytokine receptor interaction, chemokine signaling pathway, NOD-like receptor signaling pathway, and Tolllike receptor signaling pathway. Furthermore, our findings showed that SMAD3, 4 and 6 were closely associated with 
A

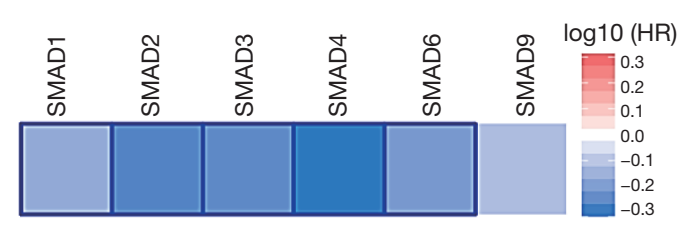

B
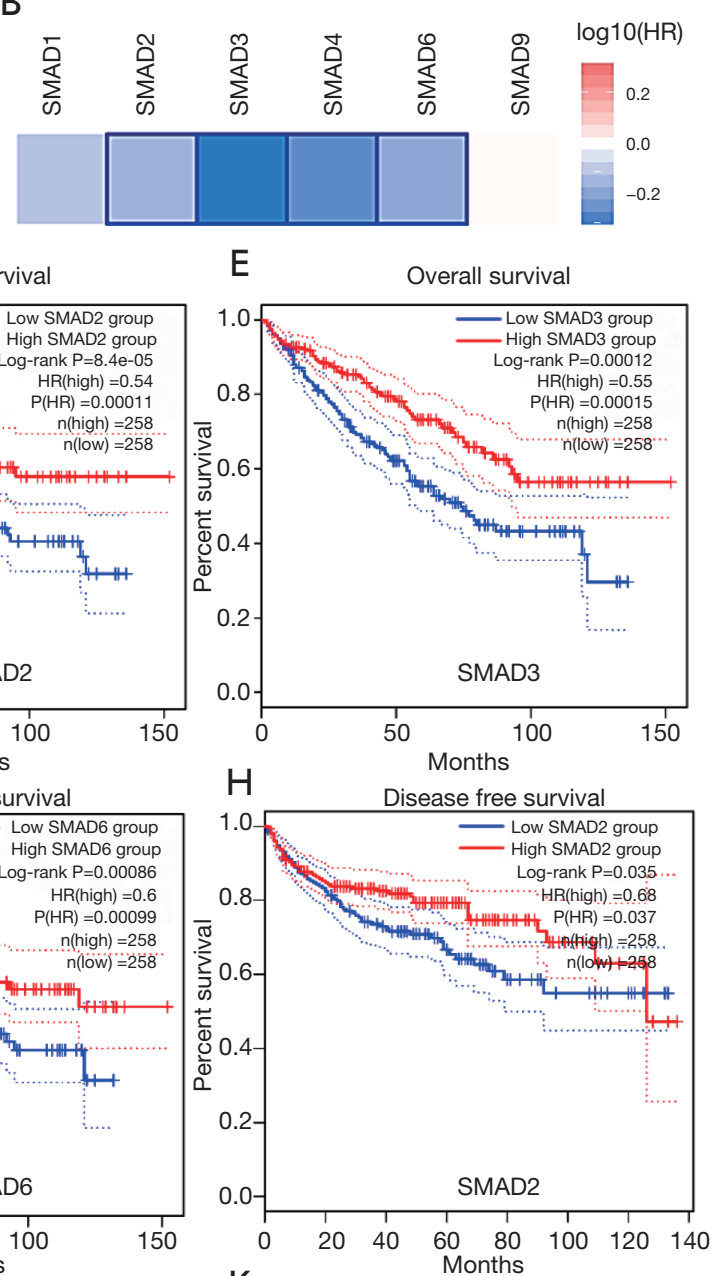

$\mathrm{K} \quad$ Disease free survival

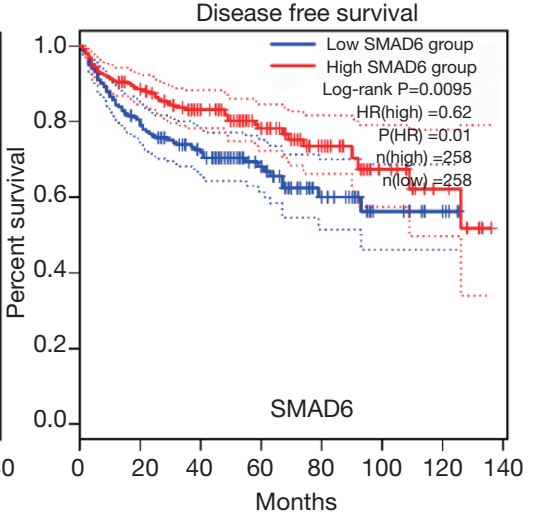

Figure 3 Prognostic value of differential expression SMAD genes in KIRC patients. (A,B) Survival significant maps of differentially expressed SMADs showed the overall survival (OS) and disease-free survival (DFS) analysis results based on the Cox proportional hazard ratio (HR) through GEPIA. The OS curve of SMAD1 (C), SMAD2 (D), SMAD3 (E), SMAD4 (F), SMAD6 (G), and the DFS curve of SMAD2 (H), SMAD3 (I), SMAD4 (J), SMAD6 (K) in KIRC. The group cutoff choice for OS was the median. A log-rank test was used to estimate the difference in OS and DFS, and a value of $\mathrm{P}<0.05$ was considered statistically significant. SMAD, small mother against decapentaplegic protein; KIRC, kidney renal clear cell carcinoma. 
A
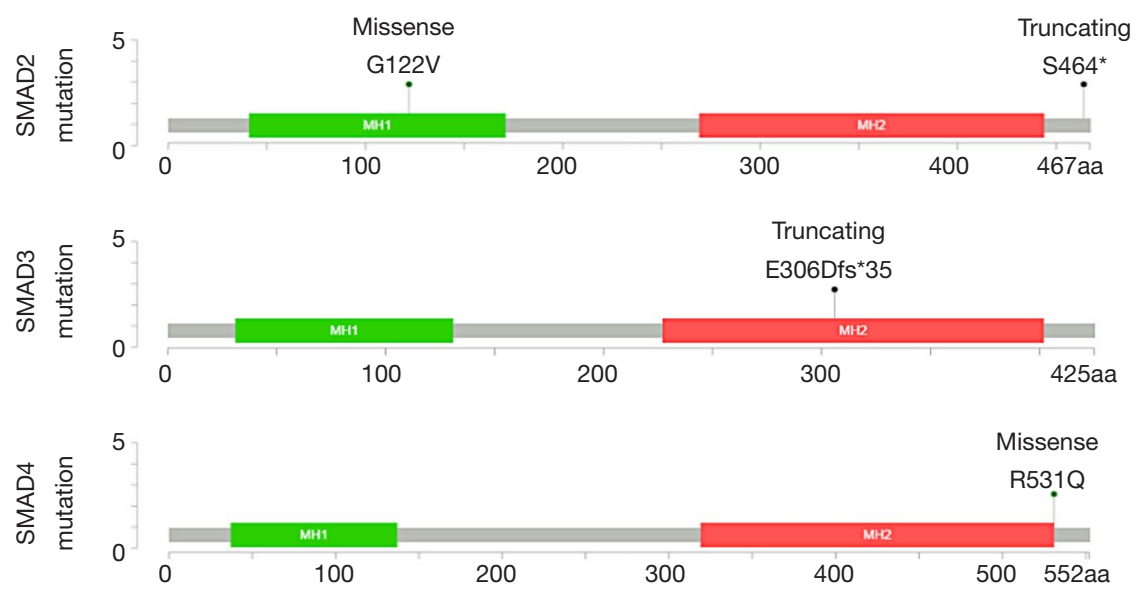

B

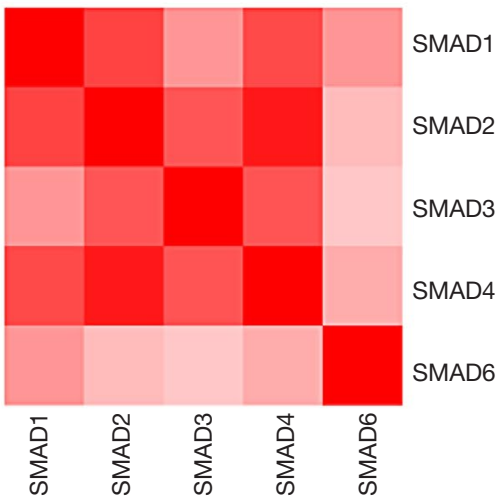

C

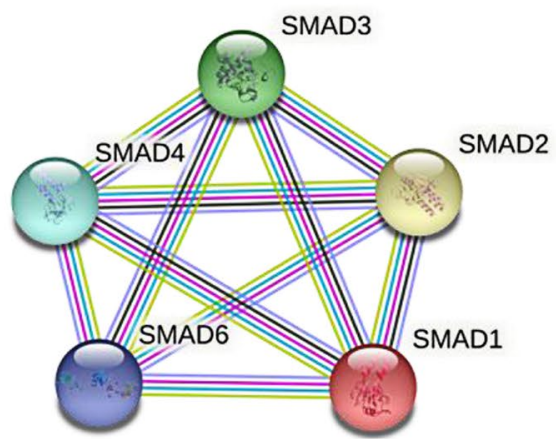

D

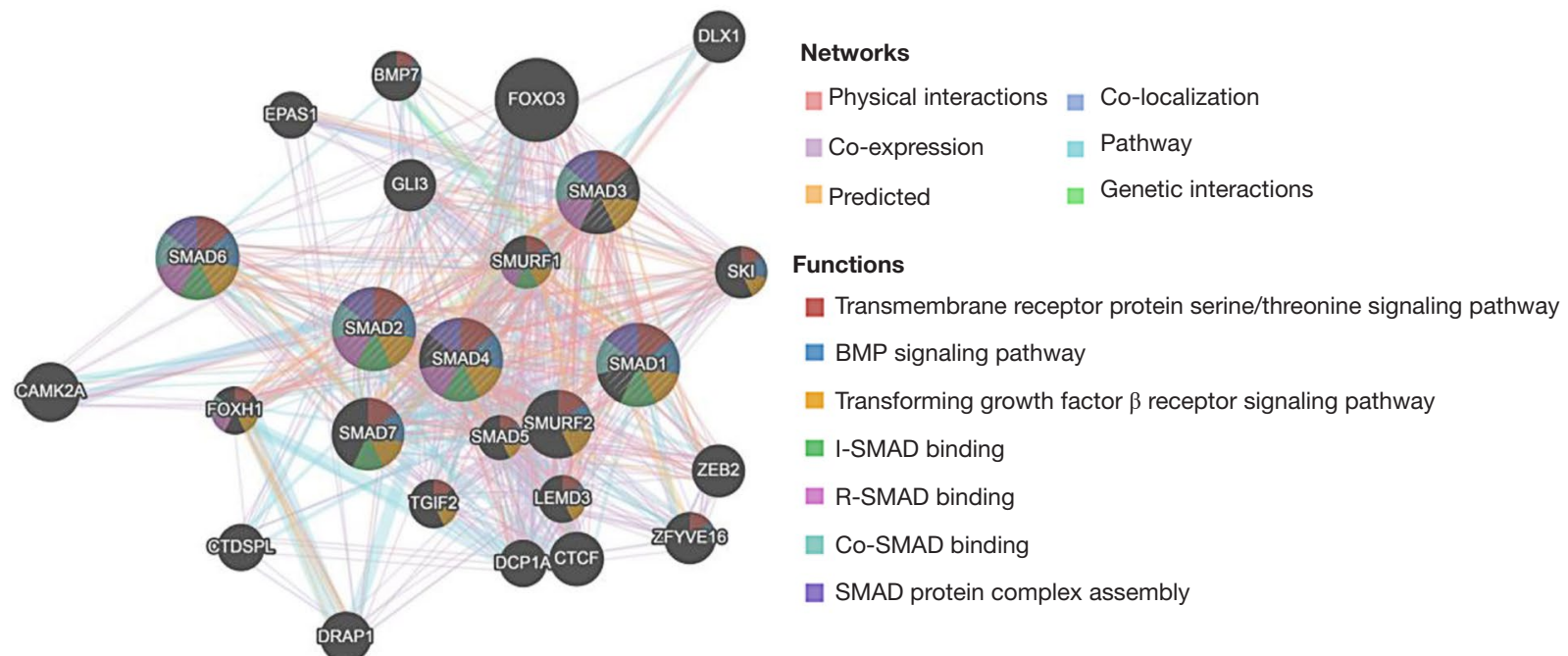

Figure 4 Genetic mutations, expression correlation, and interaction network analyses of SMAD family genes in KIRC patients. (A) Gene mutation information of SMAD genes in KIRC (cBioPortal). (B) Correlation heat map of SMAD genes in KIRC (GEPIA). (C) Protein-protein interaction network of SMAD genes (STRING). (D) The interaction network and function prediction of SMAD genes (GeneMANIA). SMAD, small mother against decapentaplegic protein; KIRC, kidney renal clear cell carcinoma. 

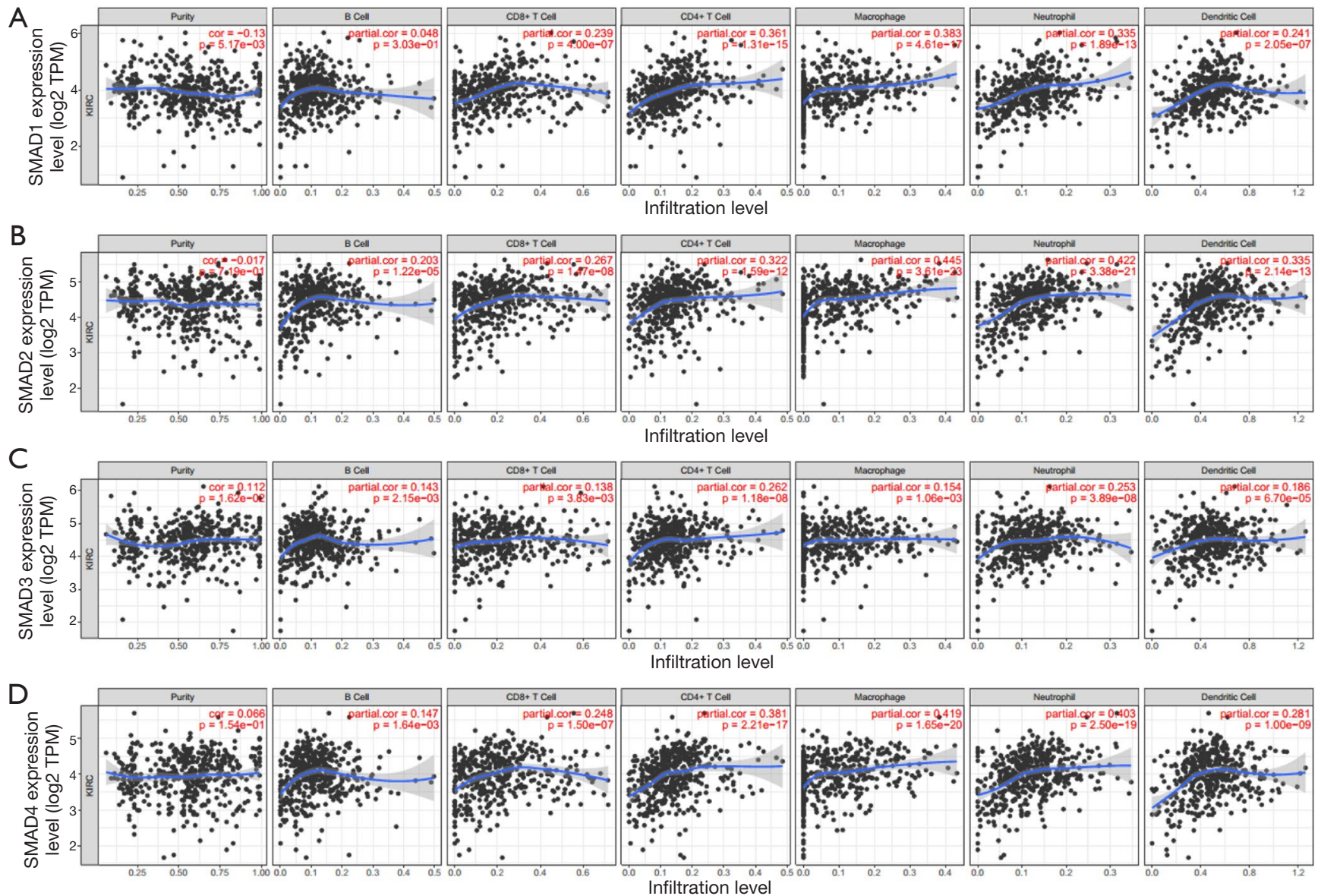

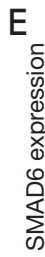
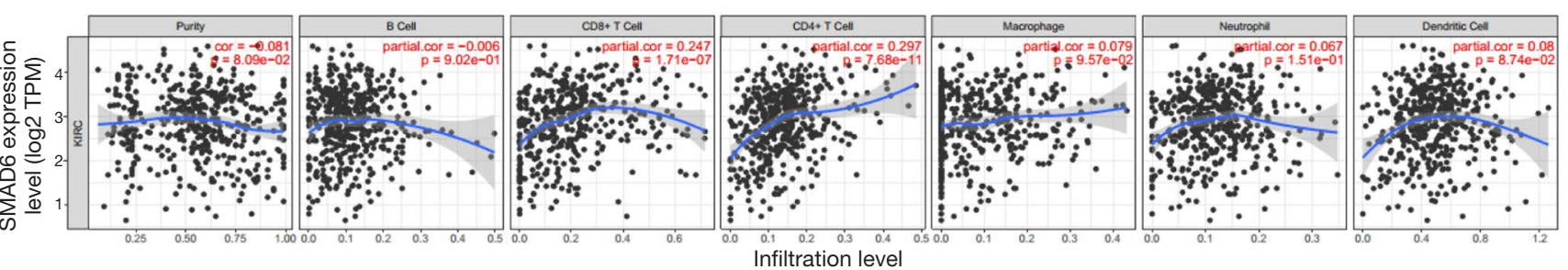

Figure 5 The expression of SMAD 1/2/3/4/6 was correlated with immune infiltration levels in patients with KIRC (TIMER). The correlation between the abundance of immune cells and the expression of SMAD1 (A), SMAD2 (B), SMAD3 (C), SMAD4 (D), SMAD6 (E) in KIRC. $\mathrm{P}<0.05$ was considered significant. SMAD, small mother against decapentaplegic protein; KIRC, kidney renal clear cell carcinoma.

the well-known pathways such as cell cycle regulation, p53 signaling pathway and JAK/STAT signaling pathway. These results implied that SMAD1, 2, 3, 4 and 6 had the potential to be therapeutic targets.

\section{Discussion}

Genetic studies have indicated that the TGF- $\beta$ /SMAD signaling pathway is involved in the regulation of tissue development and homeostasis and its dysregulation is a hallmark of many human diseases, including cancer (25). SMAD, as a family of TGF- $\beta$ signal transduction molecules, has been reported to play important roles in cell proliferation, differentiation, migration, survival and apoptosis (5). Emerging evidence has proved that SMAD family genes are abnormally expressed in various cancers, including KIRC, and may be used as prognostic biomarkers or targets of chemical drugs (26). Among these SMADs, some SMAD family genes have been well studied in various cancers. For example, SMAD2 has been confirmed to 
Table 1 Correlation analysis between SMAD1/2/3/4/6 and related markers of immune cells in TIMER

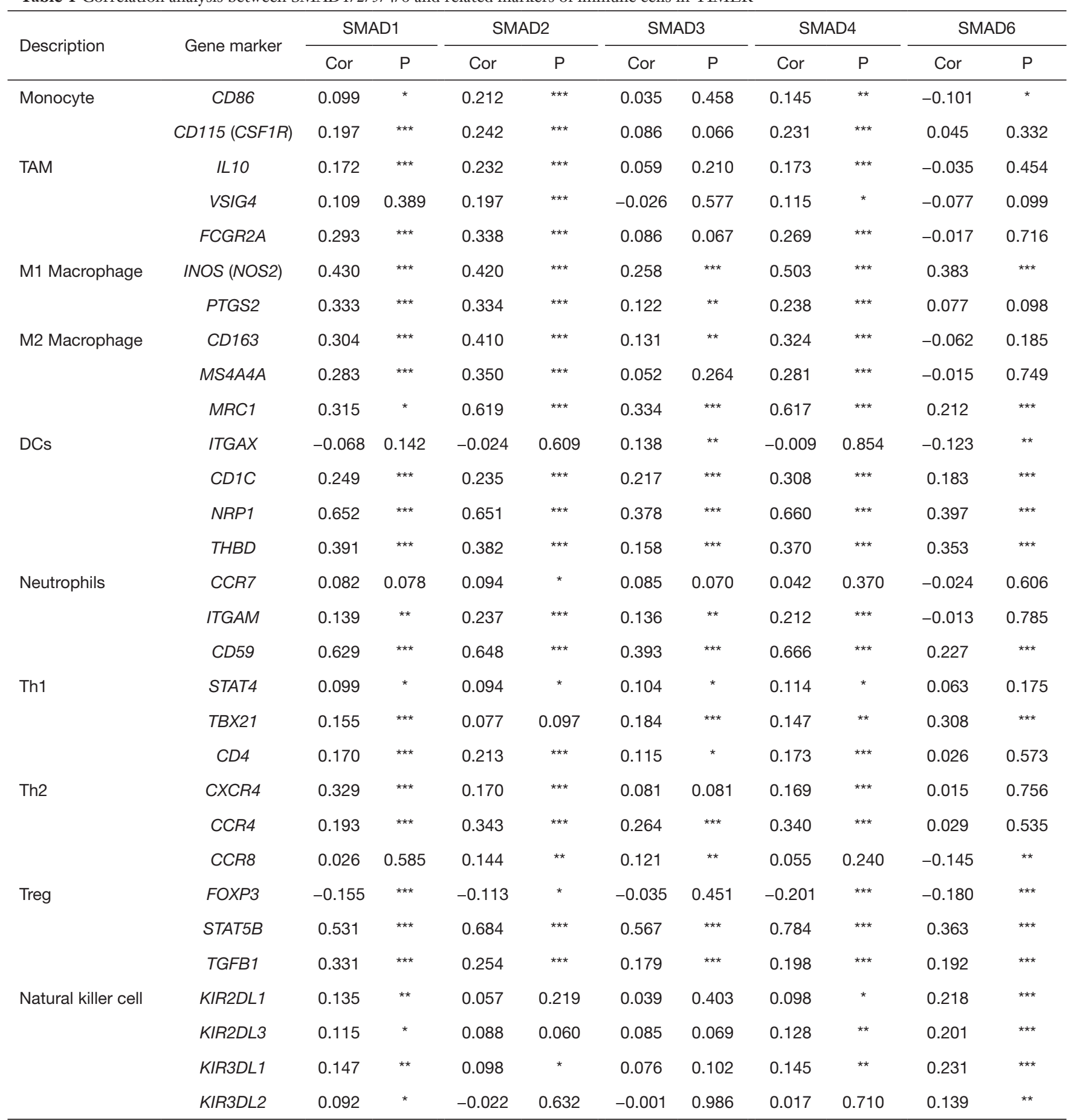

$\mathrm{P}<0.05$ was considered statistically significant $\left({ }^{*}, \mathrm{P}<0.05 ;{ }^{* *}, \mathrm{P}<0.01 ;{ }^{* \star *}, \mathrm{P}<0.001\right)$. TIMER, Tumor Immune Estimation Resource; TAM, tumor-associated macrophage; SMAD, small mother against decapentaplegic protein; KIRC, kidney renal clear cell carcinoma. 
A

C
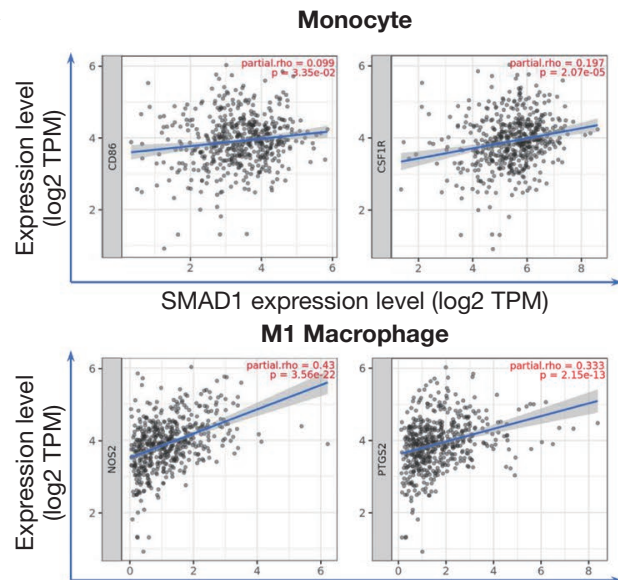

E

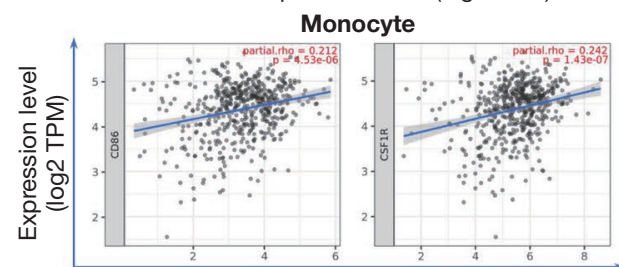

G

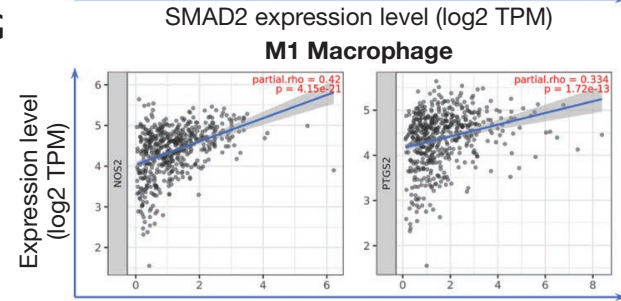

I

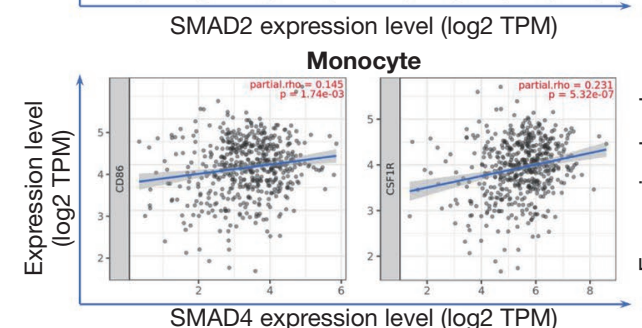

$\mathrm{K}$

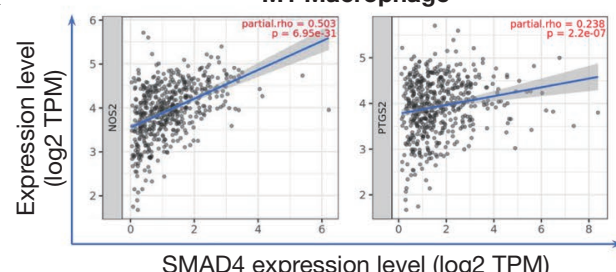

B

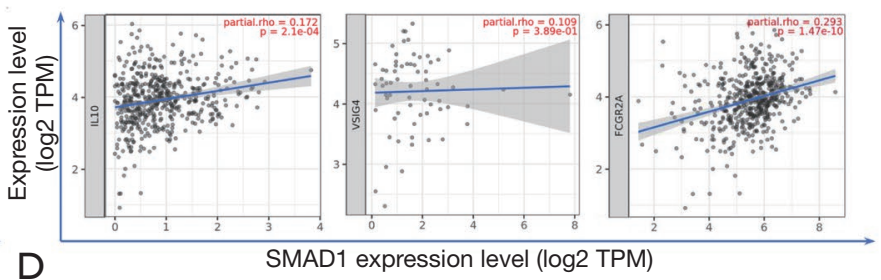

M2 Macrophage

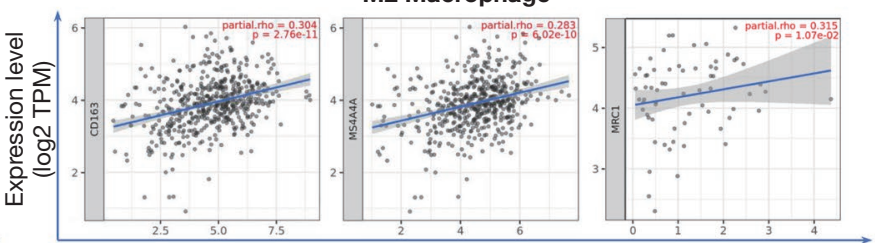

$\mathrm{F}$

SMAD1 expression level (log2 TPM)

TAM

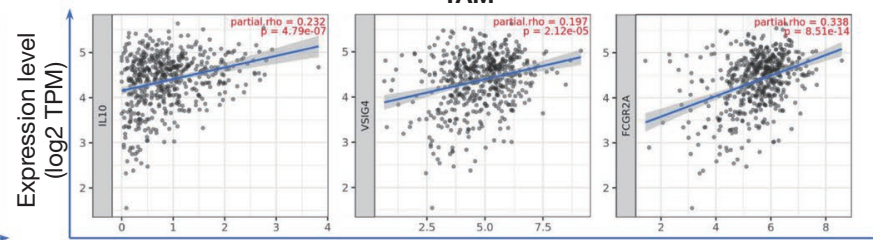

$\mathrm{H}$

SMAD2 expression level (log2 TPM)

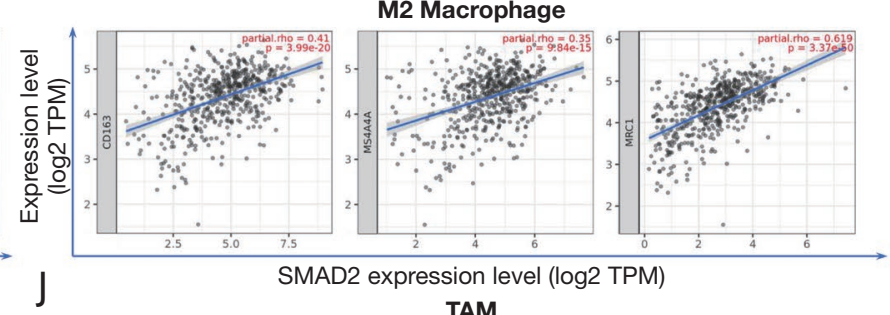

TAM

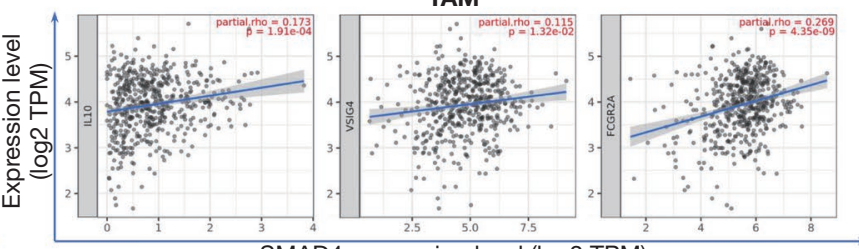

$\mathrm{L}$

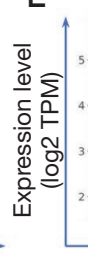

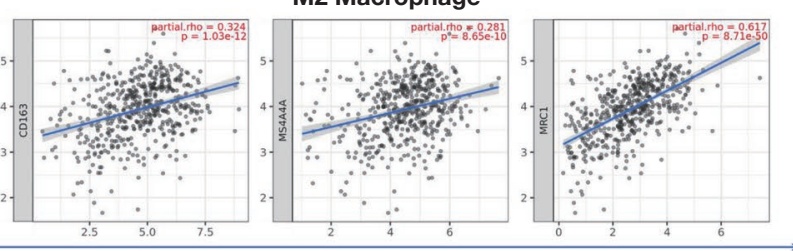

SMAD4 expression level (log2 TPM)
M2 Macrophage

Figure 6 Expressions of SMAD1/2/4 correlated with macrophage polarization in KIRC (TIMER). The correlation between the expression of SMAD1/2/4 and monocytes (gene markers: CD86 and CD115), TAM (gene markers: IL10, VSIG4 and FCGR2A), M1 macrophage (gene markers: INOS and PTGS2) and M2 macrophage (gene markers: CD163, MS4A4A and MRC1) infiltration levels was assessed. Scatterplots of correlations between SMAD1 expression and monocytes (A), TAM (B), M1 macrophage (C) and M2 macrophage (D) in KIRC. Scatterplots of correlations between SMAD2 expression and monocytes (E), TAM (F), M1 macrophage (G) and M2 macrophage (H) in KIRC. Scatterplots of correlations between SMAD4 expression and monocytes (I), TAM (J), M1 macrophage (K) and M2 macrophage (L) in KIRC. $\mathrm{P}<0.05$ was considered significant. SMAD, small mother against decapentaplegic protein; KIRC, kidney renal clear cell carcinoma. 
Table 2 Correlation analysis between SMAD1/2/4 and relate genes and markers of monocyte and macrophages in GEPIA

\begin{tabular}{|c|c|c|c|c|c|c|c|}
\hline Description & Gene marker & \multicolumn{2}{|c|}{ SMAD1 } & \multicolumn{2}{|c|}{ SMAD2 } & \multicolumn{2}{|c|}{ SMAD4 } \\
\hline \multirow[t]{2}{*}{ Monocyte } & $C D 86$ & 0.260 & $* * *$ & 0.300 & $* * *$ & 0.300 & *** \\
\hline & CD115 (CSF1R) & 0.360 & 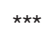 & 0.340 & $\star \star \star \star ~$ & 0.360 & *** \\
\hline \multirow[t]{2}{*}{ TAM } & IL10 & 0.320 & $* * *$ & 0.320 & $* \star *$ & 0.340 & *** \\
\hline & FCGR2A & 0.430 & $\star \star \star \star ~$ & 0.430 & 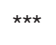 & 0.420 & *** \\
\hline \multirow[t]{2}{*}{ M1 macrophage } & INOS (NOS2) & 0.500 & $* * *$ & 0.470 & $* \star *$ & 0.490 & *** \\
\hline & PTGS2 & 0.350 & $\star \star \star \star ~$ & 0.320 & 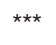 & 0.280 & *** \\
\hline M2 macrophage & $C D 163$ & 0.320 & $* \star *$ & 0.290 & $* \star \star *$ & 0.260 & *** \\
\hline
\end{tabular}

$\mathrm{P}<0.05$ was considered statistically significant $\left.{ }^{* * *}, \mathrm{P}<0.001\right)$. GEPIA, Gene Expression Profiling Interactive Analysis; TAM, tumorassociated macrophage; SMAD, small mother against decapentaplegic protein.

be associated with tumor progression and development in patients with breast cancer, gastric cancer, esophageal squamous cell carcinoma and colorectal cancer (27-30). SMAD4 played a vital role in the tumorigenesis of diverse human cancers such as squamous cell carcinoma of the esophagus, head and neck squamous cell carcinoma and pancreatic ductal adenocarcinoma, etc. (31-33). SMAD6 has been found to be a prognostic marker for patients with esophageal cancer, oral squamous cell carcinoma, and bladder cancer $(13,34,35)$. However, the prognostic value and immune infiltration relevance of SMAD family members, especially at the mRNA level, are not yet fully elucidated in KIRC.

In our study, we first explored the expression pattern of SMAD family genes in KIRC patients. We determined that the expressions of SMAD1, 2, 3, 4 and 6 were significantly reduced whereas the expression of SMAD9 was significantly increased in KIRC tissues than in normal control tissues. Further, we found that the expressions of SMAD1, 2, 3, 4 and 6 decreased considerably with the progression of KIRC tumors. Aberrant DNA methylation is almost universally found in cancer including KIRC, which can lead to silenced gene expression (36). Thus, we also explored the promoter methylation levels of these differentially expressed SMAD genes. And we found that the promoters of SMAD1, 2, 3, 4, and 6 presented higher methylation in KIRC tissues. Further, the correlation analysis displayed that the expressions of all differentially expressed SMADs were significantly and negatively correlated with their methylation levels. These results implied that aberrant methylation of these SMADs may be a primary reason for their abnormal gene expression.

Park et al. evaluated the influence of SMAD2, 3, 4 nuclear expressions on the prognosis of KIRC patients at the protein level, and found that low nuclear expressions of SMAD3 and SMAD4, but not SMAD2, were independent predictors of poor prognosis for KIRC patients (26). Consistently, we found that KIRC patients with low total expressions of the SMAD3 and SMAD4 also had a poor prognosis. On the contrary, we found that the low total expression of SMAD2 was also associated with shorter OS and DFS of KIRC patients. This may be due to the subcellular localization change of SMAD2 protein when it functions (37), so the total expression level of SMAD2 may not be correlated with their nuclear protein level. In addition, we found that the low total expressions of SMAD1 and SMAD6 were correlated with poor prognosis of KIRC patients, indicating that SMAD1 and SMAD6 had potential as prognostic markers.

Next, we evaluated the genetic mutations of SMAD family genes and found that there were missense mutations in SMAD2 and 4, and truncation mutations in SMAD2 and 3. Further, analyses of gene co-expression and PPI network demonstrated that there were a strong expression correlation and a close functional link among these differentially expressed SMAD members.

Immune infiltration of the tumor microenvironment 
A

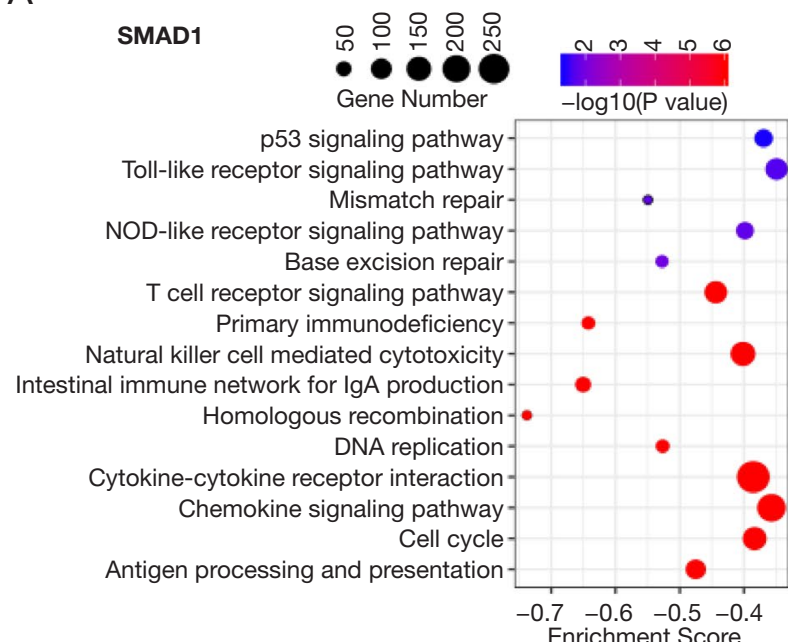

C

$E$

\section{운운 운 융 융

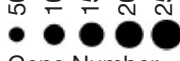 \\ Gene Number} Cell cycle

JAK-STAT signaling pathway Toll-like receptor signaling pathway.

$\mathrm{T}$ cell receptor signaling pathway

Primary immunodeficiency p53 signaling pathway

NOD-like receptor signaling pathway Natural killer cell mediated cytotoxicity . Intestinal immune network for IgA production Cytokine-cytokine receptor interaction. Chemokine signaling pathway

$B$ cell receptor signaling pathway Apoptosis

Antigen processing and presentation

$$
\begin{array}{r}
-0.7-0.6-0.5-0.4 \\
\text { Enrichment Score }
\end{array}
$$

B

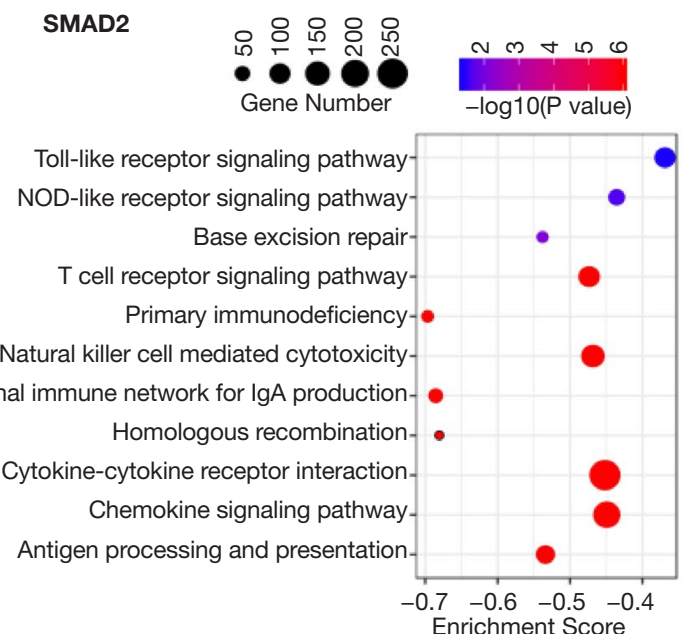

D

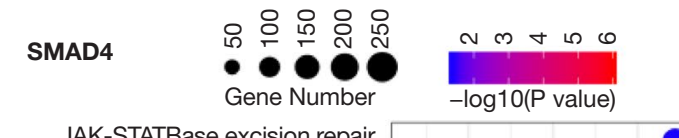

JAK-STATBase excision repair Base excision repair DNA replication Homologous recombination p53 signaling pathway Toll-like receptor signaling pathway T cell receptor signaling pathway Primary immunodeficiency NOD-like receptor signaling pathway Intestinal immune network for lgA production Cytokine-cytokine receptor interaction Chemokine signaling pathway Antigen processing and presentation
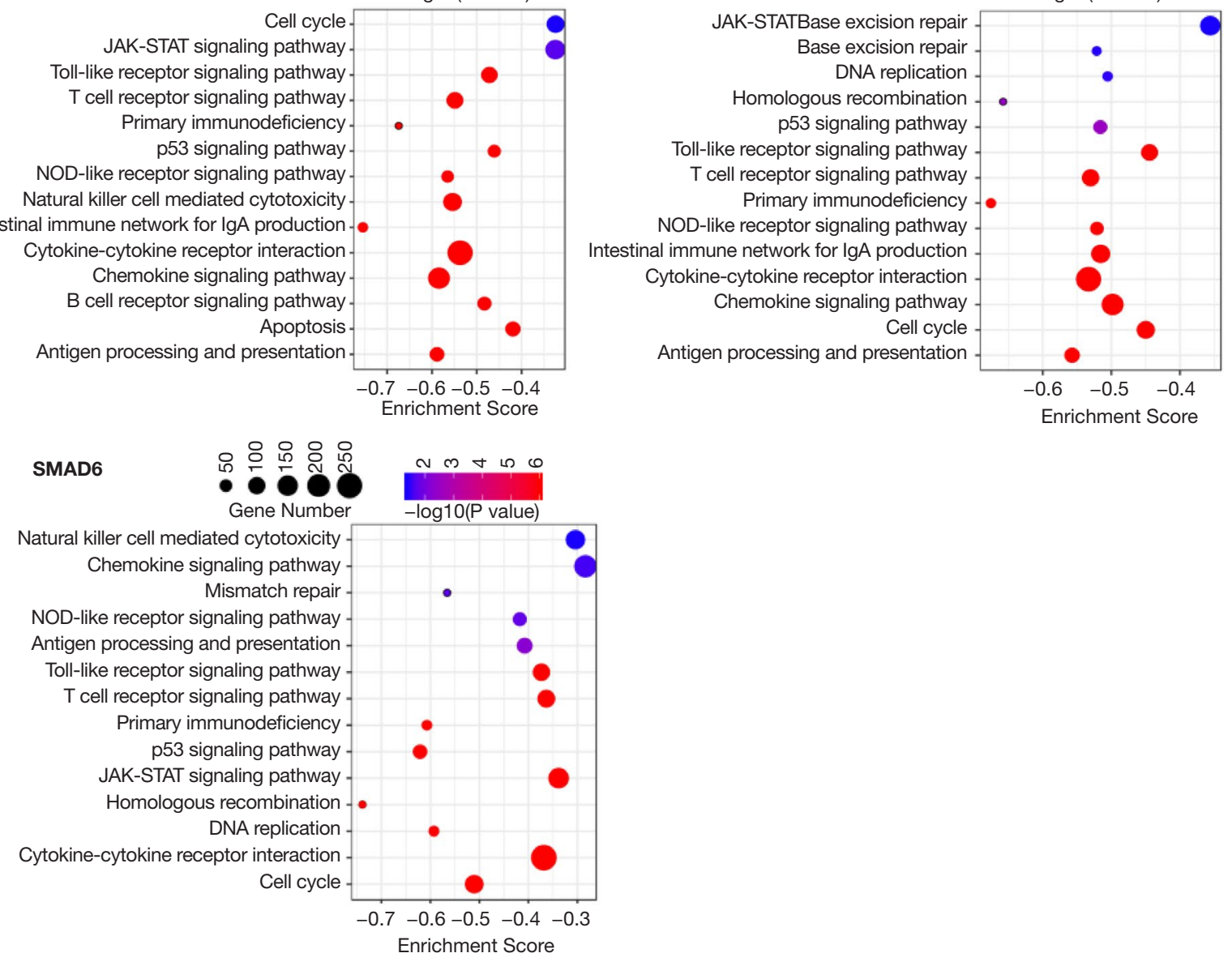

Figure 7 Functional enrichment analysis of SMAD1/2/3/4/6 in patients with KIRC. The bubble diagram displayed the GSEA results in phenotype low group of SMAD1 (A), SMAD2 (B), SMAD3 (C), SMAD4 (D), SMAD6 (E). The nominal P value $<0.05$ and false discovery rate $\mathrm{q}<0.25$ were used to screen significantly enriched gene sets. SMAD, small mother against decapentaplegic protein; KIRC, kidney renal clear cell carcinoma; GSEA, Gene Set Enrichment Analysis. 
is a crucial determining factor for immune response and is closely related to clinical prognosis in cancer including KIRC (15). Thus, we further evaluated the correlation between the differential expression of these SMADs and immune cell infiltration. In this study, we found that the expression levels of SMAD2, 3, 4 were significantly and positively related to the levels of infiltrating $\mathrm{B}$ cells, $\mathrm{CD} 4^{+} \mathrm{T}$ cells, $\mathrm{CD} 8^{+} \mathrm{T}$ cells, macrophage, neutrophils and dendritic cells. The expression of SMAD1 has a significant positive correlation with the infiltration of $\mathrm{CD}^{+} \mathrm{T}$ cells, $\mathrm{CD} 8^{+} \mathrm{T}$ cells, macrophages, neutrophils and dendritic cells. And the expression level of SMAD6 was remarkably positively correlated with the infiltrating levels of $\mathrm{CD}^{+} \mathrm{T}$ cells and $\mathrm{CD}^{+} \mathrm{T}$ cells. In addition, the correlation between SMAD1, 2, 3, 4, 6 expressions and gene expressions of immune cell markers further implicated their roles in regulating tumor immunology in KIRC. Especially, SMAD1, 2, 4 showed evident correlations with monocyte, TAM, M1/M2 macrophage, indicating that SMAD1, 2, 4 may have a vital role in regulating the polarity of macrophages. In summary, these findings indicated that SMAD1, 2, 3, 4, 6 may play an important role in the regulation and recruitment of immune infiltrating cells in KIRC.

Finally, we focused on the function of these SMADs using the GSEA method. As expected, we found that the functions of SMAD1, 2, 3, 4, 6 were relevant to immunerelated pathways such as natural killer cell-mediated cytotoxicity, primary immunodeficiency, $\mathrm{T}$ cell receptor signaling pathway, etc. They were also closely related to cytokine-cytokine receptor interaction, chemokine signaling pathway, NOD-like receptor signaling pathway, and Tolllike receptor signaling pathway. In addition, SMAD3, 4 and 6 were found to be closely associated with cell cycle regulation, p53 signaling pathway and JAK/STAT signaling pathway. These data indicated that SMAD1, 2, 3, 4, 6 can be potential therapeutic targets in KIRC.

\section{Conclusions}

We comprehensively and systematically analyzed the expression pattern, prognostic value, and immune infiltrating relevance of SMAD family genes in KIRC, and further explored their potential functions. Our results indicate that SMAD1, 2, 3, 4, 6 are potential biomarkers to predict the prognosis and immune cell infiltration of KIRC patients. We hope our results will provide novel insights to identify prognostic biomarkers, assisting the prediction of the survival of KIRC patients, and helping clinicians choose effective treatment targets for KIRC patients. However, there are certain limitations to this study. Transcription levels analysis cannot completely reflect the functional changes of genes. Thus, further in vivo and in vitro investigations are needed to verify our findings and promote a better understanding of SMADs in KIRC.

\section{Acknowledgments}

We would like to thank the service of Anhui Soula Translation for editing this manuscript.

Funding: The present study was supported by a grant from the Tianjin Major Scientific and Technological Special Project for Major Disease Prevention and Control (19ZXDBSY00090).

\section{Footnote}

Reporting Checklist: The authors have completed the REMARK reporting checklist. Available at https://dx.doi. org/10.21037/tcr-21-178

Conflicts of Interest: All authors have completed the ICMJE uniform disclosure form (available at https://dx.doi. org/10.21037/tcr-21-178). The authors have no conflicts of interest to declare.

Ethical Statement: The authors are accountable for all aspects of the work in ensuring that questions related to the accuracy or integrity of any part of the work are appropriately investigated and resolved. The study was conducted in accordance with the Declaration of Helsinki (as revised in 2013).

Open Access Statement: This is an Open Access article distributed in accordance with the Creative Commons Attribution-NonCommercial-NoDerivs 4.0 International License (CC BY-NC-ND 4.0), which permits the noncommercial replication and distribution of the article with the strict proviso that no changes or edits are made and the original work is properly cited (including links to both the formal publication through the relevant DOI and the license). See: https://creativecommons.org/licenses/by-nc-nd/4.0/.

\section{References}

1. Turajlic S, Swanton C, Boshoff C. Kidney cancer: The 
next decade. J Exp Med 2018;215:2477-9.

2. Klatte T, Rossi SH, Stewart GD. Prognostic factors and prognostic models for renal cell carcinoma: a literature review. World J Urol 2018;36:1943-52.

3. Lam JS, Shvarts O, Leppert JT, et al. Postoperative surveillance protocol for patients with localized and locally advanced renal cell carcinoma based on a validated prognostic nomogram and risk group stratification system. J Urol 2005;174:466-72; discussion 472; quiz 801.

4. Gul A, Rini BI. Adjuvant therapy in renal cell carcinoma. Cancer 2019;125:2935-44.

5. Derynck R, Zhang Y. Smad-dependent and Smadindependent pathways in TGF-beta family signalling. Nature 2003;425:577-84.

6. Macias MJ, Martin-Malpartida P, Massagué J. Structural determinants of Smad function in TGF- $\beta$ signaling. Trends Biochem Sci 2015;40:296-308.

7. Huminiecki L, Goldovsky L, Freilich S, et al. Emergence, development and diversification of the TGF-beta signalling pathway within the animal kingdom. BMC Evol Biol 2009;9:28.

8. Meng XM, Chung AC, Lan HY. Role of the TGF- $\beta /$ BMP-7/Smad pathways in renal diseases. Clin Sci (Lond) 2013;124:243-54.

9. Zeng Z, Yang Y, Qing C, et al. Distinct expression and prognostic value of members of SMAD family in nonsmall cell lung cancer. Medicine 2020;99:e19451.

10. Zhang $X$, Feng $W$, Zhang J, et al. Long non-coding RNA PVT1 promotes epithelial-mesenchymal transition via the TGF- $\beta /$ Smad pathway in pancreatic cancer cells. Oncol Rep 2018;40:1093-102.

11. Chandrasinghe P, Cereser B, Moorghen M, et al. Role of SMAD proteins in colitis-associated cancer: from known to the unknown. Oncogene 2018;37:1-7.

12. Kim YH, Lee HS, Lee HJ, et al. Prognostic significance of the expression of Smad4 and Smad7 in human gastric carcinomas. Ann Oncol 2004; 15:574-80.

13. Osawa H, Nakajima M, Kato H, et al. Prognostic value of the expression of Smad6 and Smad7, as inhibitory Smads of the TGF-beta superfamily, in esophageal squamous cell carcinoma. Anticancer Res 2004;24:3703-9.

14. Ino Y, Yamazaki-Itoh R, Shimada $\mathrm{K}$, et al. Immune cell infiltration as an indicator of the immune microenvironment of pancreatic cancer. Br J Cancer 2013;108:914-23.

15. Zhang S, Zhang E, Long J, et al. Immune infiltration in renal cell carcinoma. Cancer Sci 2019;110:1564-72.

16. Mahmoud SM, Paish EC, Powe DG, et al. Tumor- infiltrating CD8+ lymphocytes predict clinical outcome in breast cancer. J Clin Oncol 2011;29:1949-55.

17. Grivennikov SI, Greten FR, Karin M. Immunity, inflammation, and cancer. Cell 2010;140:883-99.

18. Ross K, Jones RJ. Immune checkpoint inhibitors in renal cell carcinoma. Clin Sci (Lond) 2017;131:2627-42.

19. Chandrashekar DS, Bashel B, Balasubramanya SAH, et al. UALCAN: A Portal for Facilitating Tumor Subgroup Gene Expression and Survival Analyses. Neoplasia 2017;19:649-58.

20. Vasaikar SV, Straub P, Wang J, et al. LinkedOmics: analyzing multi-omics data within and across 32 cancer types. Nucleic Acids Res 2018;46:D956-63.

21. Tang Z, Li C, Kang B, Gao G, et al. GEPIA: a web server for cancer and normal gene expression profiling and interactive analyses. Nucleic Acids Res 2017;45:W98-W102.

22. Li T, Fan J, Wang B, et al. TIMER: A Web Server for Comprehensive Analysis of Tumor-Infiltrating Immune Cells. Cancer Res 2017;77:e108-10.

23. Subramanian A, Tamayo P, Mootha VK, et al. Gene set enrichment analysis: a knowledge-based approach for interpreting genome-wide expression profiles. Proc Natl Acad Sci U S A 2005;102:15545-50.

24. Morris MR, Maher ER. Epigenetics of renal cell carcinoma: the path towards new diagnostics and therapeutics. Genome Med 2010;2:59.

25. ten Dijke P, Arthur HM. Extracellular control of TGFbeta signalling in vascular development and disease. Nat Rev Mol Cell Biol 2007;8:857-69.

26. Park JH, Lee C, Suh JH, et al. Nuclear expression of Smad proteins and its prognostic significance in clear cell renal cell carcinoma. Hum Pathol 2013;44:2047-54.

27. Xie W, Mertens JC, Reiss DJ, et al. Alterations of Smad signaling in human breast carcinoma are associated with poor outcome: a tissue microarray study. Cancer Res 2002;62:497-505.

28. Shinto O, Yashiro M, Toyokawa T, et al. Phosphorylated smad2 in advanced stage gastric carcinoma. BMC Cancer 2010;10:652.

29. Xie W, Rimm DL, Lin Y, et al. Loss of Smad signaling in human colorectal cancer is associated with advanced disease and poor prognosis. Cancer J 2003;9:302-12.

30. Fukuchi M, Nakajima M, Miyazaki T, et al. Lack of activated Smad2 in transforming growth factor-beta signaling is an unfavorable prognostic factor in patients with esophageal squamous cell carcinoma. J Surg Oncol 2006;94:51-6. 
31. Bardeesy N, Cheng KH, Berger JH, et al. Smad4 is dispensable for normal pancreas development yet critical in progression and tumor biology of pancreas cancer. Genes Dev 2006;20:3130-46.

32. Korc M. Smad4: gatekeeper gene in head and neck squamous cell carcinoma. J Clin Invest 2009;119:3208-11.

33. Natsugoe S, Xiangming C, Matsumoto M, et al. Smad4 and transforming growth factor beta1 expression in patients with squamous cell carcinoma of the esophagus. Clin Cancer Res 2002;8:1838-42.

34. Mangone FR, Walder F, Maistro S, et al. Smad2 and Smad6 as predictors of overall survival in oral squamous

Cite this article as: Zhang S, Liu Z, Xiao M, Liu H. The prognostic value and immunological role of the small mother against decapentaplegic proteins in kidney renal clear cell carcinoma. Transl Cancer Res 2021;10(6):2678-2693. doi: $10.21037 /$ tcr-21-178 cell carcinoma patients. Mol Cancer 2010;9:106.

35. Riester M, Taylor JM, Feifer A, et al. Combination of a novel gene expression signature with a clinical nomogram improves the prediction of survival in high-risk bladder cancer. Clin Cancer Res 2012;18:1323-33.

36. Xu L, He J, Cai Q, et al. An effective seven-CpG-based signature to predict survival in renal clear cell carcinoma by integrating DNA methylation and gene expression. Life Sci 2020;243:117289.

37. Cheng J, Grande JP. Transforming growth factor-beta signal transduction and progressive renal disease. Exp Biol Med (Maywood) 2002;227:943-56. 